\title{
Antimicrobial Resistance of Streptococcus uberis Isolated from Bovine Mastitis: A Review
}

\author{
Tingrui Zhang ${ }^{1}$, Linli Tao², Sukolrat Boonyayatra ${ }^{3}$, Guoyi Niu²
}

10.18805/IJAR.B-1377

\begin{abstract}
Bovine mastitis is one of the common diseases resulting in high economic losses in the dairy industry. Streptococcus uberis, the environmental or contagious pathogen, is one of the most frequently identified bacteria causing clinical and subclinical mastitis. Antimicrobials are commonly used to control bacterial infections in dairy cattle. The emergence of antimicrobial resistance (AMR) bacteria made the treatment of this disease by antimicrobials a challenge. Currently, AMR is a global threat to both human and animal health. This review summarizes the AMR profiles of S. uberis collected worldwide between the years 2000-2020. Most of the studies included in this review were from Europe, Estonia, Canada, Danish, Switzerland and Czech. In general, S. uberis is highly susceptible to $\beta$-lactam antimicrobials, whereas resistance to tetracyclines, macrolides, aminoglycosides antimicrobials occurred in most countries. The isolates against most antimicrobials presented an increasing pattern over time. It highlights that monitoring the AMR of $S$. uberis is crucial to reduce the public health crisis.
\end{abstract}

Key words: Antimicrobial resistance, Bovine mastitis, Streptococcus uberis.

Mastitis is a major infectious disease of dairy cattle throughout the world, which consisted of clinical mastitis and subclinical mastitis. It leads to losses associated with the reduction of milk production and quality, treatment cost, milk discard and animal mortality (Bianchi et al. 2019; Saravanan et al. 2015). Clinical mastitis shows several clinical signs including abnormal udder size and milk secretion, reduced milk production, whereas subclinical mastitis may not show any signs on the udder or changes in the milk (Ashraf et al. 2018; Sharma et al. 2012). Enormous economic losses caused by mastitis was observed worldwide (Bianchi et al. 2019; Kappeli et al. 2019). In US dairy, the total cost in the average case of clinical mastitis was $\$ 444$ (Rollin et al. 2015). An annual loss of EUR 9.03 billion associated with summer mastitis was reported in the UK dairy industry (Halasa et al. 2007).

Mastitis pathogens can be divided into two groups including contagious and environmental pathogens. Streptococcus uberis has been identified as the most frequently isolated environmental or contagious pathogen from clinical and subclinical mastitis (Cameron et al. 2016; Wente et al. 2019). It can colonize animals and their environment without the need for a specific living environment such as udder milk. Previous research reported that $51.6 \%$ and $63-85 \%$ of $S$. uberis were isolated from the skin of dairy cows and environmental samples including water, soil and bedding materials, respectively (Kromker, 2014). S. uberis can produce biofilms that might result in persistent intramammary infections and treatment failure through increased resistance to antibiotics (Schönborn et al. 2017). Thus, S. uberis, the difficult-to-handle pathogen, was considered as the barrier to the control of bovine mastitis as the epidemiology was not completely understood (Tomazi et al. 2019; Wente et al. 2019).
${ }^{1}$ Graduate Program in Veterinary Science, Faculty of Veterinary Medicine, Chiang Mai University, Chiang Mai, Thailand.

${ }^{2}$ Faculty of Animal Science and Technology, Yunnan Agricultural University, Kunming, P.R China.

${ }^{3}$ Department of Food Animal Clinic, Faculty of Veterinary Medicine, Chiang Mai University, Chiang Mai, Thailand.

Corresponding Author: Guoyi Niu, Faculty of Animal Science and Technology, Yunnan Agricultural University, Kunming, P.R China. Email: nguoyi@163.com

How to cite this article: Zhang, T., Tao, L., Boonyayatra, S. and Niu, G. (2021). Antimicrobial Resistance of Streptococcus uberis Isolated from Bovine Mastitis: A Review. Indian Journal of Animal Research. DOI: 10.18805/IJAR.B-1377.

Submitted: 22-04-2021 Accepted: 02-11-2021 Online: 25-12-2021

Antimicrobial usage is the most effective method for treating mastitis caused by bacteria. Antimicrobial treatment was reported in most cases of mastitis worldwide (Song et al. 2020; Wente et al. 2019). However, the treatment of environmental and contagious pathogens of $S$. uberis presents a challenge for the management of dairy cattle (Tian et al. 2019). The overuse and misuse of antimicrobials play a significant role in increasing resistance to them. The emergence of resistant isolates occurred in the gene mutation or horizontal transmission of antimicrobial resistance genes (ARG) from another microorganism (McDougall et al. 2020). In recent decades, resistance to antimicrobial agents has become a global problem for both human and animal health. Antimicrobial resistance (AMR) in dairy cattle can impact humans due to the potential dissemination of AMR pathogens to humans via consumption of infected dairy products or contact with infected dairy cattle (Molineri et al. 2021). Additionally, animal 
waste with resistant isolates and antimicrobials could impact the environment. Therefore, monitoring the antimicrobial susceptibility of $S$. uberis is essential to guide the veterinaries in selecting the most appropriate antimicrobials for treating the disease. The most frequently used antimicrobial classes for treating bovine mastitis caused by S. uberis are $\beta$-lactam, aminoglycosides, tetracyclines and macrolides (EI Garch et al. 2020; Loch et al. 2005; McDougall et al. 2020). This review aimed to describe the phenotypic resistance to antimicrobials in $S$. uberis collected in worldwide between 2000-2020.

\section{Prevalence of pathogens}

A variety of pathogens can cause mastitis in dairy cows, the most common contagious mastitis pathogens were Staphylococcus aureus and Streptococcus agalactiae (Juozaitienë et al. 2020; Shome et al. 2012). In South America, S. agalactiae showed 35\% frequencies (Kabelitz et al. 2021). S. aureus was isolated from $51.2 \%$ mastitis milk samples from Ethiopia (Abebe et al. 2016), whereas $10.17-16.67 \%$ of $S$ aureus was detected in Finland (Pitkälä et al. 2004). However, several studies have shown that the most prevalent pathogens causing clinical mastitis in cows are usually organisms that originate from the environment. Common environmental organisms include coagulasenegative staphylococci (CNS), S. uberis, Streptococcus dysgalactiae, Klebsiella spp. and Escherichia coli (Sharma et al. 2012). The research reported in Wisconsin showed that the most prevalent pathogens were E. coli $(22.5 \%)$, followed by environmental streptococci (12.8\%), Klebsiella spp. (6.9\%) and CNS (6.1\%) (Oliveira et al. 2013). Song et al. (2020) reported that the most frequently isolated pathogens from mastitis milk samples were environmental bacteria (67.53\%). S. agalactiae and S. uberis were more prevalent in farms using sand bedding. Environmental streptococci, especially $S$. uberis ranks among the prime causative agents of mastitis around the world. Mastitis caused by $S$. uberis showed an increasing trend in dairy farms (Kromker. 2014). Thus, this review aimed to summarize the antimicrobial susceptibility of $S$. uberis.

\section{Antimicrobial resistance}

\section{Resistance to $\beta$-lactams}

$S$. uberis has historically been regarded as highly susceptible to $\beta$-lactam antimicrobials because it didn't produce $\beta$-lactamase. Thus, these antimicrobials were the first choice for this disease infected by the bacteria. In general, published data revealed that $S$. uberis was highly susceptible to $\beta$-lactam antimicrobials including ampicillin, penicillin $\mathrm{G}$, amoxicillin, ceftiofur, oxacillin, cloxacillin (Table 1). However, the ongoing pan-European antimicrobial susceptibility monitoring programme (VetPath) in Europe reported increased median minimum inhibitory concentrations $\left(\mathrm{MIC}_{50}\right.$ ) value for penicillin $\mathrm{G}$ in $S$. uberis from 0.03 to 0.12 during 2002 to 2012 (de Jong et al., 2018; Thomas et al. 2015). Increased MIC of oxacillin in S. uberis in New Zealand was observed as well (McDougall et al. 2014). In addition, phenotypical resistance to penicillin was isolated from Estonia (0.4\%), New Zealand (1\%), Slovakia $(10.5 \%)$, Canada (5.8\%), Switzerland $(7.7 \%)$ and Korea (8.1\%). High levels of resistance to oxacillin were observed in Switzerland (64.7\%) and Korea (33.3\%). AMR of S. uberis strains vary among studies due to various elements including the sample selection and size, the population of isolates, the time and place of the study, the methods of susceptibility test, the breakpoints and references used and the analyses carried out. Meeting all these criteria makes studies comparison difficult. However, this review can provide useful references to address the problem.Penicillin-binding proteins (PBPs) are important proteins involved in the construction of cell wall peptidoglycan. Resistance against $\beta$-lactam antimicrobials, especially for gram-positive cocci, was mainly caused by altered PBPs. McDougall et al. (2020) investigated the genetic basis of increasing MIC for $\beta$-lactam of $S$. uberis. The results indicated that the presence of $p b p$ substitutions was associated with decreased susceptibility to $\beta$-lactam antimicrobials and lower cure rate outcomes following antimicrobial therapy for clinical mastitis. Similarly, the presence of $\beta$-lactamase enzymes was frequent with high MIC values of $\beta$-lactam antimicrobials for $S$. uberis isolates (Velez et al. 2017). However, Kaczorek et al. (2017) reported no relationship between blaZ and increased MIC values in Poland. Therefore, more research is needed to determine the mechanism of $\beta$-lactam resistance of $S$. uberis.

\section{Resistance to macrolides}

Macrolides are commonly used for the treatment of bovine mastitis caused by streptococci. Previously, the frequency of resistance to macrolides for streptococci was less than $10 \%$ in dairy cattle (Loch et al. 2005). S. uberis was highly susceptible to erythromycin. Although erythromycin resistance frequency varied in different studies, most frequencies were $1.9 \%-24 \%$ and the highest resistance frequency (34.3\%) was observed by Nam et al. (2009) in Korea.

Resistance to macrolides can be caused by several mechanisms such as the presence of ribosomal methylase genes [erm (A), erm (B), erm (C)] and efflux pump genes [mef (A), mef (E), msr (A)] (Rossolini et al. 2017). The erm genes are currently the most common functional gene responsible for the resistance to macrolides observed in streptococci (Entorf et al. 2016). The erm(B) was most frequently detected among erythromycin-resistant streptococci (Entorf et al. 2016), which in accordance with the finding in France (Haenni et al. 2011). According to Loch et al. (2005) all $S$. uberis isolates with MIC $\geq 16$ for erythromycin were positive for erm(B) gene. Similarly, Kaczorek (2017) reported that the erm(B) gene predominates in Streptococcus spp. Previous research demonstrated that it might be due to the horizontal gene transfer among the bacteria of genus Streptococcus from cattle or farm environments (Loch et al. 2005). 


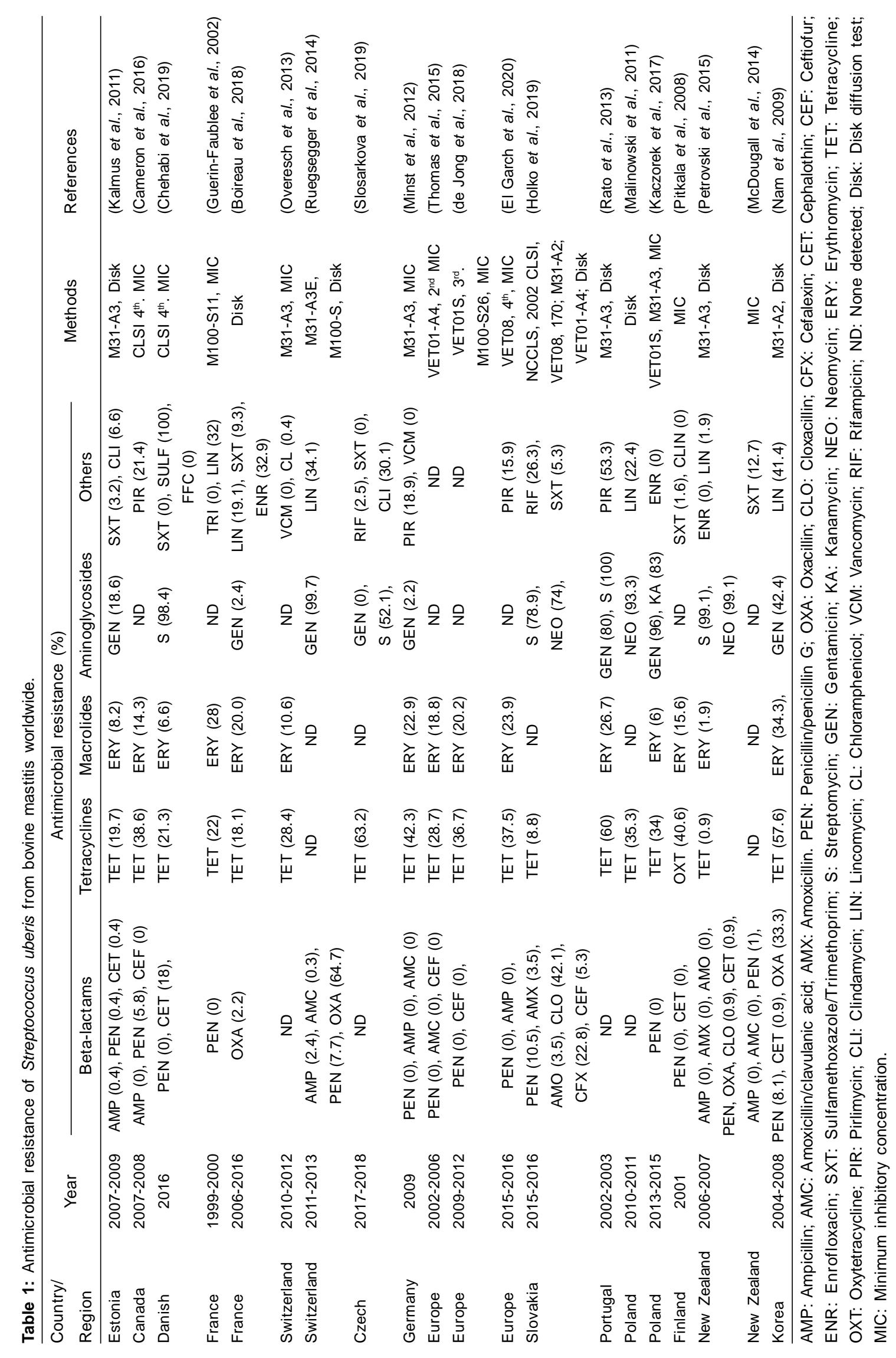




\section{Resistance to tetracyclines}

Tetracycline is a broad-spectrum antimicrobial against a wide variety of gram-positive and gram-negative bacteria. It is extensively used in animals, which is also important for treating the same bacteria in humans. Thus, it is not surprising the less effective antimicrobial therapy caused by the resistant pathogens in dairy cattle. Resistance to tetracycline varied in different studies. Several studies have reported that high levels of resistance to tetracycline were found in most countries including Estonia (19.7\%), Canada (38.6\%), Danish $(21.3 \%)$, France $(18.1 \%)$, Switzerland (28.4\%), Czech (63.2\%), Germany (42.3\%), Portugal (60\%), Poland (35.3\%) and Korea (57.6\%), whereas resistance to tetracycline was $0.9 \%$ in New Zealand and $8.8 \%$ in Slovakia. Additionally, resistance trend to tetracycline was linear increase from $15.7 \%$ in January 2006 to $20.4 \%$ in December 2016 in France (Boireau et al. 2018). A similar trend was found in Europe (de Jong et al. 2018; El Garch et al. 2020; Thomas et al. 2015). Resistance to tetracyclines in Streptococcus spp. can be due to several different mechanism such as ribosomal protection [tet $(\mathrm{M})$, tet $(\mathrm{O})$ ], tetracycline efflux system [tet $(\mathrm{L})$, tet $(\mathrm{K})$, tet (40)] (Rossolini et al. 2017). However, limited research based on the resistance mechanism in $S$. uberis was reported.

\section{Resistance to aminoglycosides}

Aminoglycosides may be used to the treatment of bovine mastitis. The present data showed that $S$. uberis was highly resistant to aminoglycosides antimicrobials. Resistance to gentamicin showed a broad range (0-99.7\%), whereas higher level of resistance to streptomycin was found in all published research. The research observed by Kaczorek et al. (2017) indicated that Streptococcus spp. showed naturally low effective against aminoglycosides antimicrobials.

\section{Resistance to other antimicrobials}

Several studies reported the resistance to sulfamethoxazole/ trimethoprim in Estonia (3.2\%), France (9.3\%), Slovakia $(5.3 \%)$, Finland (1.6\%) and New Zealand (12.7\%). Pirlimycin was used to treat bovine mastitis caused by gram-positive cocci, which is approved for veterinary use (El Garch et al. 2020). Resistance to pirlimycin was detected in Canada $(21.4 \%)$, Germany (18.9\%), Europe (15.9\%) and Portugal (53.3\%). The study observed by Pol et al. (2007) indicated that increased MIC of pirlimycin was associated with increasing exposure to defined daily doses of antimicrobial.

\section{Antimicrobial resistance over time}

This review reported the phenotypic resistance to antimicrobials in $S$. uberis collected from worldwide between 2000-2020. In this period, the resistance trend to tetracycline was linear increase from $15.7 \%$ in January 2006 to $20.4 \%$ in December 2016 in France (Boireau et al. 2018). Similarly, the prevalence of AMR to tetracycline in Europe presented an increase trend from $28.7 \%$ in 2002 to $37.5 \%$ in 2016 (Fig 1). In Poland, the results showed a similar prevalence of AMR to tetracycline from 2010 to 2015 . However, it is difficult to

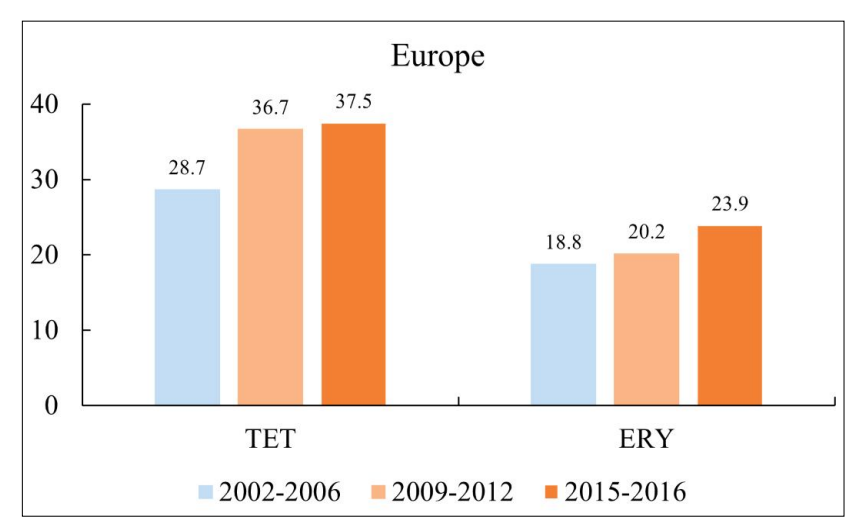

Fig 1: Trends of antimicrobial resistance for Streptococcus uberis during the 2002-2016 period in Europe.

compare as different methods were used in the research. For erythromycin, the AMR prevalence in Europe was increased from $18.8 \%$ to $23.9 \%$ during 2002 to 2016 .

The development of AMR is considered a problem to public health. According to the findings in this review, the increased AMR resistance for several antibiotics was detected as antibiotic usage in dairy farms. However, most research could not reflect the trend because test method, sampling and sample size, statistical analysis may influence the results.

Monitoring of antimicrobial usage and AMR in bacteria isolated from food-producing animals are essential for determining the emergence of resistant bacteria and providing strategies to reduce the spread of resistance. This article reviewed the AMR of $S$. uberis associated with bovine mastitis worldwide. It is difficult to compare the AMR patterns among countries as several factors may affect the results including differences in the methods performed in various research (disc diffusion or MIC), lack of interpretive criteria, differences in sample collection (El Garch et al. 2020; Molineri et al. 2021). Systematic review and meta-analysis reported by Molineri et al. (2021) demonstrated that studies using disc diffusion method presented higher AMR of Staphylococcus aureus than those using MIC. The AMR prevalence of cefoxitin, erythromycin, gentamycin and oxacillin was $10.57,5.47,3.94$ and 2.94 times higher for disc diffusion method than for MIC, respectively. Currently, only a few clinical breakpoints were used for antimicrobials against $S$. uberis. Methodology should be improved for surveillance data and optimize mastitis treatment. Additionally, most research on AMR of $S$. uberis was reported in Europe according to the present review (Fig 2). More surveillance is needed in other regions.

Antimicrobials are commonly used to treat bovine mastitis. The development of AMR is associated with antimicrobials usage in dairy farms. However, few studies investigated its relationship and only a few studies have compared the trends in AMR of $S$. uberis from the same geographical area and method (Boireau et al. 2018; de Jong et al. 2018; El Garch et al. 2020; Thomas et al. 2015). It is necessary to continuously monitor the AMR of bovine 


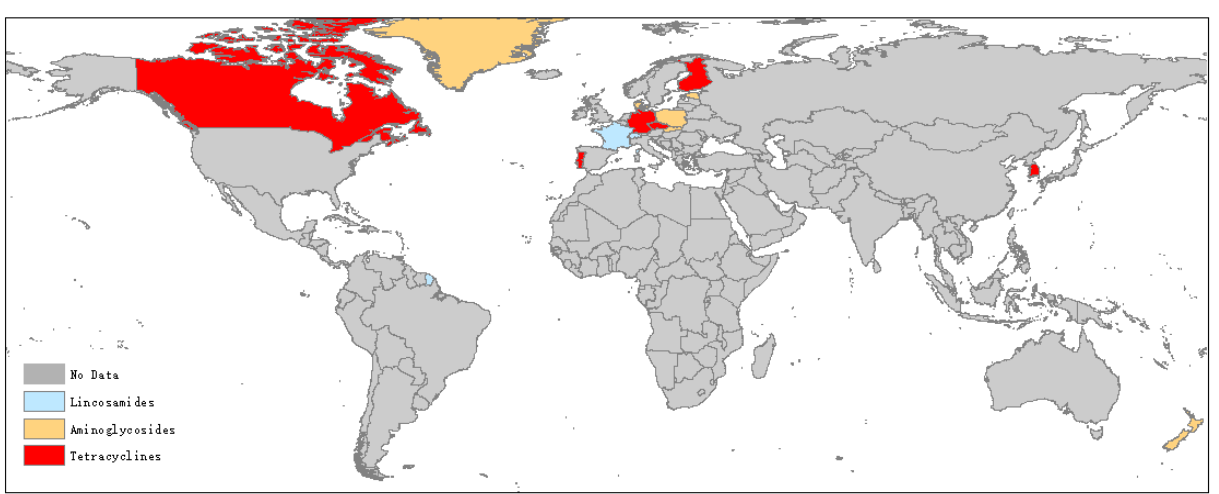

Fig 2: Distribution of antimicrobial resistance of Streptococcus uberis worldwide in this review.

mastitis pathogens worldwide and guide the veterinarians or farms to improve the management practices regarding antimicrobials usage.

Prevention of mastitis in dairy cows is better than treatment. The vaccine is considered an effective measure to prevent bacterial mastitis. However, a vaccine that prevents $S$. uberis mastitis is not available. Therefore, the control and prevention measures need to target the relevant infection risks, for example, poor environmental hygiene or non-use of teat-sealants in the dry period, bedding materials, dirty udders (Klaas et al. 2018). The incidence of environmental streptococcal mastitis in the dry period is 5.5 times higher than in other breastfeeding periods. Farmers commonly use antimicrobials to treat it in the dry period. The basis of all prevention measures for streptococcal mastitis is to limit the exposure of the nipple ends. Therefore, the lying and bedding area should be kept clean, especially dry and avoid placing too deep straw bags. Sand or sawdust is preferred (Hillerton et al. 2003). It is recommended that cows stand for at least one hour after milking until the teat canal is properly closed. In addition, the infected cattle should be isolated to avoid the spread of bacteria in the herd. Good milking operations such as teat disinfection and drying, regular cleaning and inspection of the milking machine, could reduce the bacterial contamination of individual cows. The application of these measures has reduced the incidence of streptococcal mastitis by $50 \%$ (Hogan et al. 2003).

\section{CONCLUSION}

The present review concludes the antimicrobial resistance of $S$. uberis associated with bovine mastitis worldwide. In general, $S$. uberis is highly susceptible to beta-lactam antimicrobials, whereas resistance to tetracyclines, macrolides, aminoglycosides antimicrobials occurred in most countries, which may be harmful to both animal and human health. This review has important implications for the control of $S$. uberis in clinical therapy. These findings emphasize the importance of constantly monitoring the use of antimicrobials and AMR of $S$. uberis in the world. It is also necessary to explore the resistance mechanism to antimicrobials of $S$. uberis. Farm management, mastitis surveillance and prevention programs should be improved to reduce the overall use of antimicrobials and public health threats.

\section{REFERENCES}

Abebe, R., Hatiya, H., Abera, M., Megersa, B., and Asmare, K. (2016). Bovine mastitis: Prevalence, risk factors and isolation of Staphylococcus aureus in dairy herds at Hawassa milk shed, South Ethiopia. BMC Veterinary Research. 12(1): 270. https://doi.org/10.1186/s12917-016-0905-3.

Ashraf, A., Imran, M. (2018). Diagnosis of bovine mastitis: From laboratory to farm. Tropical Animal Health and Production. 50(6): 1193-1202.

Bianchi, R.M., Schwertz, C.I., de Cecco, B.S., Panziera, W., De Lorenzo, C., Heck, L.C., Snel, G.G.M., Lopes, B.C., da Silva, F.S., Pavarini, S.P., Driemeier, D. (2019). Pathological and microbiological characterization of mastitis in dairy cows. Tropical Animal Health and Production. 51(7): 2057-2066.

Boireau, C., Cazeau, G., Jarrige, N., Calavas, D., Madec, J.Y., Leblond, A., Haenni, M., Gay, E. (2018). Antimicrobial resistance in bacteria isolated from mastitis in dairy cattle in France, 2006-2016. Journal of Dairy Science. 101(10): 9451-9462.

Cameron, M., Saab, M., Heider, L., McClure, J.T., RodriguezLecompte, J.C., Sanchez, J. (2016). Antimicrobial susceptibility patterns of environmental streptococci recovered from bovine milk samples in the Maritime Provinces of Canada. Frontiers in Veterinary Science. 3: 79. doi: $10.3389 / f v e t s .2016 .00079$

Chehabi, C.N., Nonnemann, B., Astrup, L.B., Farre, M., Pedersen, K. (2019). In vitro antimicrobial resistance of causative agents to clinical mastitis in danish dairy cows. Foodborne Pathogens and Disease. 16(8): 562-572.

de Jong, A., El Garch, F., Simjee, S., Moyaert, H., Rose, M., Youala, M., Siegwart, E., VetPath Study, G. (2018). Monitoring of antimicrobial susceptibility of udder pathogens recovered from cases of clinical mastitis in dairy cows across Europe: VetPath results. Veterinary Microbiology. 213: 73-81.

El Garch, F., Youala, M., Simjee, S., Moyaert, H., Klee, R., Truszkowska, B., Rose, M., Hocquet, D., Valot, B., Morrissey, I., de Jong, A. (2020). Antimicrobial susceptibility of nine udder pathogens recovered from bovine clinical mastitis milk in Europe 2015-2016: VetPath results. Veterinary Microbiology. 245: 108644. https://doi.org/10.1016/ j.vetmic.2020.108644. 
Entorf, M., Fessler, A.T., Kaspar, H., Kadlec, K., Peters, T., Schwarz, S. (2016). Comparative erythromycin and tylosin susceptibility testing of streptococci from bovine mastitis. Veterinary Microbiology. 194: 36-42.

Guerin-Faublee, V., Tardy, F., Bouveron, C., Carret, G. (2002). Antimicrobial susceptibility of Streptococcus species isolated from clinical mastitis in dairy cows. International Journal of Antimicrobial Agents. 19(3): 219-226.

Haenni, M., Saras, E., Chaussiere, S., Treilles, M., Madec, J Y. (2011). ermB-mediated erythromycin resistance in Streptococcus uberis from bovine mastitis. Veterinary Journal. 189(3): 356-358.

Halasa, T., Huijps, K., Østerås, O., Hogeveen, H. (2007). Economic effects of bovine mastitis and mastitis management: $\mathrm{A}$ review. Veterinary Quarterly. 29(1): 18-31.

Hillerton, J.E. and Berry, E.A. (2003). The management and treatment of environmental streptococcal mastitis. Veterinary Clinics of North America: Food Animal Practice. 19(1): 157-169.

Hogan, J. and Smith, K. (2003). Environmental streptococcal mastitis: Facts, fables and fallacies. Paper presented at the Fifth International Dairy Housing Conference for 2003.

Holko, I., Tancin, V., Vrskova, M., Tvarozkova, K. (2019). Prevalence and antimicrobial susceptibility of udder pathogens isolated from dairy cows in Slovakia. Journal of Dairy Research. 86(4): 436-439.

Juozaitienë, V., Anskienë, L., Čereğkienë, E., Japertienë, R., Şymantienë, J., Rupauskas, M. et al. (2020). Association of milk flow curve and prevalence of mastitis pathogens in dairy cows. Indian Journal of Animal Research. 54(9): 1155-1158.

Kabelitz, T., Aubry, E., Van Vorst, K., Amon, T. and Fulde, M. (2021). The role of Streptococcus spp. in bovine mastitis. Microorganisms. 9(7): 1497.

Kaczorek, E., Malaczewska, J., Wojcik, R., Rekawek, W., Siwicki, A.K. (2017). Phenotypic and genotypic antimicrobial susceptibility pattern of Streptococcus spp. isolated from cases of clinical mastitis in dairy cattle in Poland. Journal of Dairy Science. 100(8): 6442-6453.

Kalmus, P., Aasmae, B., Karssin, A., Orro, T., Kask, K. (2011). Udder pathogens and their resistance to antimicrobial agents in dairy cows in Estonia. Acta Veterinaria Scandinavica. 53.

Kappeli, N., Morach, M., Zurfluh, K., Corti, S., Nuesch-Inderbinen, M., Stephan, R. (2019). Sequence types and antimicrobial resistance profiles of Streptococcus uberis isolated from bovine mastitis. Frontiers in Veterinary Science. 6: 234.

Klaas, I.C. and Zadoks, R.N. (2018). An update on environmental mastitis: Challenging perceptions. Transboundary and Emerging Diseases. 65(S1): 166-185.

Kromker, V. (2014). Bovine Streptococcus uberis intramammary infections and mastitis. Clinical Microbiology: Open Access, 03(04).

Loch, I.M., Glenn, K., Zadoks, R.N. (2005). Macrolide and lincosamide resistance genes of environmental streptococci from bovine milk. Veterinary Microbiology. 111(1-2): 133-138.

Malinowski, E., Lassa, H., Gajewski, Z. (2011). Antibiotic Sensitivity of Streptococci and Enterococci from mastitis in cows 2010-2011. Bulletin of the Veterinary Institute in Pulawy. 55(4): 669-675.
McDougall, S., Clausen, L., Ha, H J., Gibson, I., Bryan, M., Hadjirin, N., Lay, E., Raisen, C., Ba, X., Restif, O., Parkhill, J., Holmes, M.A. (2020). Mechanisms of beta-lactam resistance of Streptococcus uberis isolated from bovine mastitis cases. Veterinary Microbiology. 242: 108592.

McDougall, S., Hussein, H., Petrovski, K. (2014). Antimicrobial resistance in Staphylococcus aureus, Streptococcus uberis and Streptococcus dysgalactiae from dairy cows with mastitis. New Zealand Veterinary Journal. 62(2): 68-76.

Minst, K., Martlbauer, E., Miller, T., Meyer, C. (2012). Short communication: Streptococcus species isolated from mastitis milk samples in Germany and their resistance to antimicrobial agents. Journal of Dairy Science. 95(12): 6957-6962.

Molineri, A I., Camussone, C., Zbrun, M V., Suarez Archilla, G., Cristiani, M., Neder, V., Calvinho, L., Signorini, M. (2021). Antimicrobial resistance of Staphylococcus aureus isolated from bovine mastitis: Systematic review and meta-analysis. Preventive Veterinary Medicine. 188: 105261.

Nam, H.M., Lim, S.K., Kang, H.M., Kim, J.M., Moon, J.S., Jang, K.C., Joo, Y.S., Kang, M.I., Jung, S.C. (2009). Antimicrobial resistance of Streptococci isolated from mastitic bovine milk samples in Korea. Journal of Veterinary Diagnostic Investigation. 21(5): 698-701.

Oliveira, L., Hulland, C., Ruegg, P.L. (2013). Characterization of clinical mastitis occurring in cows on 50 large dairy herds in Wisconsin. Journal of Dairy Science 96: 7538-7549.

Overesch, G., Stephan, R., Perreten, V. (2013). Antimicrobial susceptibility of gram-positive udder pathogens from bovine mastitis milk in Switzerland. Schweizer Archiv. Fur. Tierheilkunde. 155(6): 339-350.

Petrovski, K.R., Grinberg, A., Williamson, N.B., Abdalla, M.E., LopezVillalobos, N., Parkinson, T.J., Tucker, I.G., Rapnicki, P. (2015). Susceptibility to antimicrobials of mastitis-causing Staphylococcus aureus, Streptococcus uberis and Str. dysgalactiae from New Zealand and the USA as assessed by the disk diffusion test. Australian Veterinary Journal. 93(7): 227-233.

Pitkälä, A., Haveri, M., Pyörälä, S., Myllys, V. and HonkanenBuzalski, T. (2004). Bovine mastitis in Finland 2001Prevalence, distribution of bacteria and antimicrobial resistance. Journal of Dairy Science. 87(8): 2433-2441.

Pitkälä, A., Koort, J. and Björkroth, J. (2008). Identification and antimicrobial resistance of Streptococcus uberis and Streptococcus parauberis isolated from bovine milk samples. Journal of Dairy Science. 91(10): 4075-4081.

Pol, M., Ruegg, P.L. (2007). Relationship between antimicrobial drug usage and antimicrobial susceptibility of grampositive mastitis pathogens. Journal of Dairy Science. 90(1): 262-273.

Rato, M.G., Bexiga, R., Florindo, C., Cavaco, L.M., Vilela, C.L., Santos-Sanches, I. (2013). Antimicrobial resistance and molecular epidemiology of streptococci from bovine mastitis. Veterinary Microbiology. 161(3-4): 286-294.

Rollin, E., Dhuyvetter, K.C., Overton, M.W. (2015). The cost of clinical mastitis in the first 30 days of lactation: An economic modeling tool. Preventive Veterinary Medicine. 122(3): 257-264. 
Rossolini, G.M., Arena, F., Giani, T. (2017). Mechanisms of antibacterial resistance. Infectious Diseases. (pp. 1181-1196).

Ruegsegger, F., Ruf, J., Tschuor, A., Sigrist, Y., Rosskopf, M., Hassig, M. (2014). Antimicrobial susceptibility of mastitis pathogens of dairy cows in Switzerland. Schweizer Archiv. Fur. Tierheilkunde. 156(10): 483-488.

Saravanan, R., Das, D.N., De, S. and Panneerselvam, S. (2015). Effect of season and parity on somatic cell count across zebu and crossbred cattle population. Indian Journal of Animal Research. 49(3): 383-387.

Schönborn, S., Wente, N., Paduch, J H., Krömker, V. (2017). In vitro ability of mastitis causing pathogens to form biofilms. Journal of Dairy Research. 84(2): 198-201.

Sharma, A., Chhabra, R. and Sindhu, N. (2012). Prevalence of sub clinical mastitis in cows: Its etiology and antibiogram. Indian Journal of Animal Research. 46(4): 348-353.

Shome, B.R., Bhuvana, M., Mitra, S.D., Krithiga, N., Shome, R., Velu, D., Banerjee, A., Barbuddhe, S.B., Prabhudas, K., Rahman, H. (2012). Molecular characterization of Streptococcus agalactiae and Streptococcus uberis isolates from bovine milk. Tropical Animal Health and Production. 44(8): 1981-1992.

Slosarkova, S., Nedbalcova, K., Bzdil, J., Fleischer, P., Zouharova, M., Stanek, S., Kasna, E., Pechova, A. (2019). Antimicrobial Susceptibility of Streptococci most frequently isolated from Czech dairy cows with mastitis. Annals of Animal Science. 19(3): 679-694.

Song, X.B., Huang, X.P., Xu, H.Y., Zhang, C., Chen, S., Liu, F., Guan, S., Zhang, S., Zhu, K., Wu, C.M. (2020). The prevalence of pathogens causing bovine mastitis and their associated risk factors in 15 large dairy farms in China: An observational study. Veterinary Microbiology. 247: 108757. doi: 10.1016/j.vetmic.2020.108757.
Thomas, V., de Jong, A., Moyaert, H., Simjee, S., El Garch, F., Morrissey, I., Marion, H., Valle, M. (2015). Antimicrobial susceptibility monitoring of mastitis pathogens isolated from acute cases of clinical mastitis in dairy cows across Europe: VetPath results. International Journal of Antimicrobial Agents. 46(1): 13-20.

Tian, X.Y., Zheng, N., Han, R.W., Ho, H., Wang, J., Wang, Y.T., Wang, S.Q., Li, H.G., Liu, H.W., Yu, Z.N. (2019). Antimicrobial resistance and virulence genes of Streptococcus isolated from dairy cows with mastitis in China. Microbial Pathogenesis. 131: 33-39.

Tomazi, T., Freu, G., Alves, B.G., de Souza Filho, A.F., Heinemann, M.B., Veiga Dos Santos, M. (2019). Genotyping and antimicrobial resistance of Streptococcus uberis isolated from bovine clinical mastitis. PLoS One. 14(10): e0223719.

Velez, J.R., Cameron, M., Rodriguez-Lecompte, J.C., Xia, F.F., Heider, L.C., Saab, M., McClure, J.T., Sanchez, J. (2017). Whole-genome sequence analysis of antimicrobial resistance genes in Streptococcus uberis and Streptococcus dysgalactiae isolates from Canadian dairy herds. Frontiers in Veterinary Science. 4.

Wente, N., Klocke, D., Paduch, J H., Zhang, Y., Seeth, M T., ZocheGolob, V., Reinecke, F., Mohr, E., Kromker, V. (2019). Associations between Streptococcus uberis strains from the animal environment and clinical bovine mastitis cases. Journal of Dairy Science. 102(10): 9360-9369. 